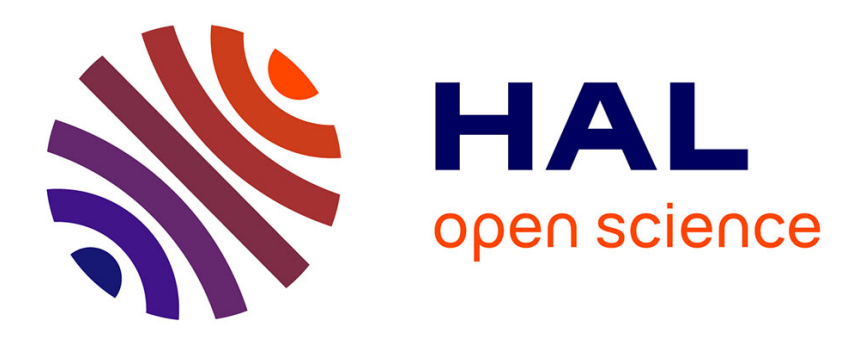

\title{
On the limit of compression of a physisorbed monolayer
}

\author{
L.W. Bruch, J.M. Gay, J. Krim
}

\section{To cite this version:}

L.W. Bruch, J.M. Gay, J. Krim. On the limit of compression of a physisorbed monolayer. Journal de Physique, 1985, 46 (3), pp.425-433. 10.1051/jphys:01985004603042500 . jpa-00209981

\section{HAL Id: jpa-00209981 https://hal.science/jpa-00209981}

Submitted on 1 Jan 1985

HAL is a multi-disciplinary open access archive for the deposit and dissemination of scientific research documents, whether they are published or not. The documents may come from teaching and research institutions in France or abroad, or from public or private research centers.
L'archive ouverte pluridisciplinaire $\mathbf{H A L}$, est destinée au dépôt et à la diffusion de documents scientifiques de niveau recherche, publiés ou non, émanant des établissements d'enseignement et de recherche français ou étrangers, des laboratoires publics ou privés. 


\title{
On the limit of compression of a physisorbed monolayer
}

\author{
L. W. Bruch $(*)$, J. M. Gay and J. Krim \\ Département de Physique (**), Faculté des Sciences de Luminy, Case 901, 13288 Marseille Cedex 9, France \\ (Reçu le 29 août 1984, accepté le 15 novembre 1984)
}

\begin{abstract}
Résumé. - On discute des effets des variations du potentiel d'interaction $\varepsilon_{0}$ entre le substrat et la première couche et de la commensurabilité uniaxiale de la monocouche sur sa limite de compression. S'il y a transition monobicouche avec seulement de faibles modifications de la distance entre proches voisins, la valeur du potentiel chimique dépend de l'interaction substrat-deuxième couche mais peu de $\varepsilon_{0}$. On discute des effets des valeurs nettement différentes de $\varepsilon_{0}$ pour divers substrats dans le cas de l'hélium. On présente des calculs sur la limite de compression du système commensurable uniaxial $\mathrm{Xe} / \mathrm{Cu}(110)$; le potentiel chimique à la transition est très proche de celui de la condensation du Xe tridimensionnel. On discute de la valeur de $\varepsilon_{0}$ pour $\mathrm{Xe} / \mathrm{Cu}(110)$ et la détermination expérimentale de la chaleur latente d'adsorption de sa monocouche.
\end{abstract}

\begin{abstract}
Effects on the limit of monolayer compression of large changes in the first layer adatom-substrate holding potential $\varepsilon_{0}$ and of uniaxial registry of the monolayer are discussed. It is shown that if the monolayerto-bilayer transition occurs with only small changes in the nearest neighbour spacing, the chemical potential there depends on the second-layer binding, but little on $\varepsilon_{0}$. Consequences of the particularly large variations in $\varepsilon_{0}$ for helium between substrates are discussed. Model calculations of the limit of compression for uniaxially registered $\mathrm{Xe} / \mathrm{Cu}(110)$ are reported; the chemical potential at the transition is quite close to that for condensation of bulk Xe. The value of $\varepsilon_{0}$ for $\mathrm{Xe} / \mathrm{Cu}(110)$ and the experimental determination of the monolayer latent heat of adsorption are discussed and a revised value is proposed for the latent heat.
\end{abstract}

\section{Introduction.}

Monolayer solids of physisorbed gases may be continuously compressed by increasing the chemical potential of the coexisting three-dimensional (3D) gas [1-6]. The limit of compression [7-10] is set by transitions to other phases and is an element of the initial stages of multilayer formation [11-13]. The theory of the monolayer-to-bilayer transition, as a limit on monolayer compression, has been developed for inert gas adsorbates ( $\mathrm{Ne}$ to $\mathrm{Xe}$ ) which have no registry with the substrate and for which the holding potential is relatively strong compared to the lateral interactions [7-10]. At low temperatures, such a monolayer-to-bilayer transition is essentially a condensation of the second atomic layer of adsorbate : at coexistence there is then only a very dilute second layer of gas above the monolayer

(*) Permanent address : Department of Physics, University of Wisconsin-Madison, Madison, Wisconsin 53706, U.S.A.

$(* *)$ Equipe de Recherche associée au Centre National de la Recherche Scientifique $\mathrm{n}^{0} 899$. solid and the bilayer consists of two solid atomic layers with very few vacancies. Here we discuss departures from the systematic trends established for the classical systems which may arise in adsorption of quantum gases [14-19] and in examples where a classical monolayer solid is in uniaxial registry with the substrate [20-22].

The monolayer (2D) solid of xenon adsorbed on the (111) face of silver, $\mathrm{Xe} / \mathrm{Ag}(111)$, displays the simplest pattern of compression $[1,7]$, with no registry phases and little effect of quantum zero-point energy. At monolayer condensation the nearest-neighbour spacing in the $2 \mathrm{D}$ solid is $2 \%$ larger than the nearestneighbour spacing $L_{\mathrm{s}}$ in the 3D solid at the sublimation curve. Under compression, the nearest-neighbour spacing decreases and at the transition to the bilayer solid, the lateral nearest-neighbour spacings in both the monolayer and bilayer are experimentally indistinguishable (equality to within $0.5 \%$ ) from $I_{\mathrm{s}}$. This pattern is observed also for $\mathrm{Kr} / \mathrm{Ag}(111)$ [1], $\mathrm{Ar} / \mathrm{Ag}(111)$ [1], and argon adsorbed on the basal plane surface of graphite, $\mathrm{Ar} / \mathrm{Gr}$ [2]. For $\mathrm{Kr} / \mathrm{Gr}$ [3], at monolayer condensation there is a registry lattice, but at the bilayer formation the nearest-neighbour spacing is 
again close to $L_{\mathrm{s}}$. For $\mathrm{Ne} / \mathrm{Gr}$ there are appreciable effects of the zero-point energy and the initial dilation of the solid monolayer is larger $[6,10]$; the limiting monolayer solid is compressed to about a $1 \%$ smaller nearest-neighbour spacing than $L_{\mathrm{s}}[5,6]$. The monolayer solid of deuterated methane on graphite, $\mathrm{CD}_{4} / \mathrm{Gr}$ [23], also follows the pattern of the classical inert gases. At $32 \mathrm{~K}$, although there is a registry lattice, the nearestneighbour spacing at the monolayer limit of compression is [24] within $0.5 \%$ of $L_{\mathrm{s}}$ [25].

The driving force of the compression in all these cases is the relatively strong holding potential for the first adsorbed layer, which makes it energetically more favorable to compress the first layer than to initiate a dense second layer. Additionally, the holding potential sets the origin of the energy scale of the multilayer phase diagram $[11,26]$ relative to the chemical potential for condensation of a dense 3D phase. However, we show in section 2 that the chemical potential $\mu_{\text {II }}$ at the condensation of the bilayer of a given adsorbate varies little for large changes in the first layer holding potential; for adsorbed helium the limiting monolayer density does vary significantly with these changes.

Structural considerations provide a guide to limited layer growth phenomena : registry, symmetry and compression each play a role [13]. The molecular packings in the first adsorbed layers may be quite different from those in the 3D solids and compressible monolayers may be driven to smaller spacings than $L_{\mathrm{s}}$ $[5,6,27]$. Registry lattices near the limit of monolayer compression $[4,20-22]$ can precede either extended or limited layer growth. For $\mathrm{Xe} / \mathrm{Gr}$ there are temperatures where a registry lattice at the monolayer limit is followed [4] by a bilayer solid with spacing close to $L_{\mathrm{s}}$ and then with an extended layer-by-layer growth of the $\mathrm{Xe}[12]$. For uniaxial registry lattices of $\mathrm{Xe}$ on $\mathrm{Ag}(110)$ and $\mathrm{Cu}(110)$, reported for the monolayer regime $[20,21]$, there is only limited experimental information [22] on the growth beyond the monolayer solid; our model calculation for $\mathrm{Xe} / \mathrm{Cu}(110)$ in section 3 shows it to be a marginal case for second-layer growth.

The organization of this paper is : in section 2 we discuss the classical pattern of the monolayer-tobilayer transition, relating the insensitivity of the chemical potential $\mu_{\text {II }}$ at the transition under changes of first-layer holding potential $\varepsilon_{0}$ to the small (or zero) lattice constant discontinuities at the transition. Some estimates for the behaviour of compressed monolayers of helium, based on binding energies for single adatoms derived from atom-surface scattering experiments [14-19], are also given there. In section 3 we present our model calculation for the limit of compression of $\mathrm{Xe} / \mathrm{Cu}(110)$, based on a first-layer interaction model constructed previously [28]. We propose an increase of the value for the monolayer latent heat of condensation of $\mathrm{Xe} / \mathrm{Cu}(110)$ to make the calculations consistent with the observed compressions [21]. In section 4 we present some concluding remarks.

\section{A classical pattern of adsorption.}

For $\mathrm{Xe}$, as for $\mathrm{Kr}$, the first layer holding potentials on $\mathrm{Gr}$ and on $\mathrm{Ag}(111)$ are quite close in magnitude [29]. However, for $\mathrm{He}$ [15-17] and for $\mathrm{H}_{2}$ [17, 18], the holding potentials on noble metals are quite different than on basal plane graphite $[14,19]$. The lowest bound state energy $\left(\varepsilon_{0}\right)$ of ${ }^{4} \mathrm{He} / \mathrm{Gr}$ is $12.1 \mathrm{meV}$ [14], while for the noble metals the values are : $4.5 \mathrm{meV}$ for ${ }^{4} \mathrm{He} / \mathrm{Ag}(110)$ [16], $6 \mathrm{meV}$ for ${ }^{4} \mathrm{He} / \mathrm{Au}(110)$ [15] and $4.6 \mathrm{meV}$ for ${ }^{4} \mathrm{He} / \mathrm{Cu}(113)$ [17]. However, the coefficient $\left(C_{3}\right)$ of the van der Waals attraction to the substrate [30], which sets the scale of the interaction $\left(\varepsilon_{\mathrm{II}}\right)$ of second-layer adatoms with the substrate, is some $50 \%$ larger for these metals than for $\mathrm{He} / \mathrm{Gr}$. Thus there is a large contrast in $\varepsilon_{0}-\varepsilon_{\mathrm{II}}$ for $\mathrm{Gr}$ and for noble metals. In this section we discuss the effects of such large changes in the holding potential on the monolayerto-bilayer transition, using a zero temperature formalism previously applied to near-classical adsorbates [7]. We also comment on the system ${ }^{3} \mathrm{He} / \mathrm{H}_{2}$ for which the $\varepsilon_{0}$ is approximately $1 \mathrm{meV}[31,32]$.

In addition to the adatom/substrate energies $\varepsilon_{0}$ and $\varepsilon_{\mathrm{II}}$ introduced in the preceding paragraph, we denote the adatom-adatom contributions to the internal energy of the monolayer and bilayer by $\varepsilon_{f 1}$ and $\varepsilon_{\ell 2}$. The potential energies for $\varepsilon_{\rho_{1}}, \varepsilon_{\ell_{2}}$ and $\varepsilon_{I I}$ in the case of classical adlayers are displayed in equations (3.8) and (3.11). The internal energy per adatom of the monolayer is

$$
u_{1}=\varepsilon_{0}+\varepsilon_{\ell 1}
$$

and for the bilayer it is

$$
u_{2}=\frac{1}{2}\left(\varepsilon_{0}+\varepsilon_{\mathrm{II}}+\varepsilon_{\ell 2}\right) .
$$

Denoting the areas per adatom in the monolayer and bilayer stacks by $a_{1}$ and $a_{2}$, the zero temperature spreading pressures and chemical potentials (enthalpies) are

$$
\phi_{i}=-\mathrm{d} u_{i} / \mathrm{d} a_{i} \quad i=1,2 .
$$

At the monolayer-to-bilayer transition the conditions of mechanical and mass transfer equilibrium are

$$
\begin{aligned}
& \phi_{1}=\phi_{2} \\
& \mu_{1}=\mu_{2}=\mu_{\mathrm{II}}
\end{aligned}
$$

so that the chemical potential is

$$
\begin{aligned}
\mu_{\mathrm{II}}=u_{1}- & a_{1} \frac{\left(u_{1}-u_{2}\right)}{\left(a_{1}-a_{2}\right)} \\
= & {\left[1-\frac{1}{2} \frac{a_{1}}{a_{1}-a_{2}}\right]\left(\varepsilon_{0}+\varepsilon_{l 1}\right)+} \\
& \quad+\frac{1}{2} \frac{a_{1}}{a_{1}-a_{2}}\left(\varepsilon_{\mathrm{II}}+\varepsilon_{l 2}-\varepsilon_{\ell_{1}}\right) .
\end{aligned}
$$


The chemical potential at monolayer condensation for a self-bound layer is

$$
\mu_{\mathrm{I}}=\varepsilon_{0}+\varepsilon_{p_{1}}(\mathrm{~min}) .
$$

For near-classical adsorbates, without registry effects, there has been no observed discontinuity in the lateral nearest-neighbour spacing at the monolayerto-bilayer transition [1-6]. This result can be expressed as

$$
a_{1} / a_{2} \simeq 2
$$

and $\mu_{\mathrm{II}}$ is then

$$
\mu_{\mathrm{II}}(\text { classical }) \simeq \varepsilon_{\mathrm{II}}+\left(\varepsilon_{\ell 2}-\varepsilon_{\ell 1}\right) .
$$

For $\mathrm{Xe}, \mathrm{Kr}$, and $\mathrm{Ar}$ on $\mathrm{Ag}(111)$ the experimental limit on the difference between the lateral nearneighbour spacings of the coexisting bilayer and monolayer is $0.01 \AA[1]$; then equation (2.7) is fulfilled to $0.5 \%$ and the correction to equation (2.8) is of order $0.005 \varepsilon_{0}(10 \mathrm{~K}$ for $\mathrm{Xe})$. For $\mathrm{Xe} / \mathrm{Gr}$, at $60 \mathrm{~K}$ there is [4] a $2.5 \%$ difference in the spacings at coexistence and a $5 \%$ correction to equation (7); the corresponding corrections to equation (2.8) would include approximately $100 \mathrm{~K}$ from the first term on the right-hand side of equation (2.5) and a nearly offsetting correction (in this case) from the second term on the right-hand side of equation (2.5).

Equation (2.8) shows that when equation (2.7) is fulfilled the chemical potential at bilayer condensation is insensitive to the magnitude of $\varepsilon_{0}$, but varies directly with $C_{3}$ (see Eq. (3.8)). While the energies $\varepsilon_{p_{i}}$ vary rapidly with lateral spacing, the net change in $\varepsilon_{\ell_{1}}$ of a near-classical monolayer for the rather small lattice compressions between $\mu_{\mathrm{I}}$ and $\mu_{\mathrm{II}}$ tends to be small. Further, for the classical adlayers where the energies are sums of adatom interaction potentials, the effects of substrate-mediated interactions among the first layer of adatoms largely cancel in the difference $\varepsilon_{\ell 1}-\varepsilon_{\ell 2}$, as can be seen by inspecting the way the first-layer lateral potential energy $\Phi_{11}$ enters in equations (3.11) for a classical adlayer. Thus the chemical potential at second-layer condensation $\mu_{\mathrm{II}}$, relative to the chemical potential $\mu_{0}$ for bulk condensation, is only weakly dependent on substrate processes involving first-layer adatoms [30]. This analysis can be extended to the bilayer-to-trilayer solid transition, which was shown in a model calculation for a classical adlayer [7] to have a smaller lattice constant discontinuity than the corresponding monolayer-to-bilayer transition. These conditions approximate the conditions assumed in the construction of the SingletonHalsey theory of multilayer adsorption [33].

We show data for the monolayer and bilayer condensations of $\mathrm{Xe} / \mathrm{Ag}(111), \mathrm{Xe} / \mathrm{Gr}$, and $\mathrm{Xe} / \mathrm{Pd}(100)$ $[1,4,34,35]$ and the extrapolation of bulk sublimation [36] in figure 1. For $\mathrm{Xe} / \mathrm{Ag}(111)$ and $\mathrm{Xe} / \mathrm{Gr}$ the monolayer condensations nearly coincide; the slightly higher pressures for the $\mathrm{Xe} / \mathrm{Gr}$ bilayer may reflect both the difference in $C_{3}$ and the small lattice constant discon-

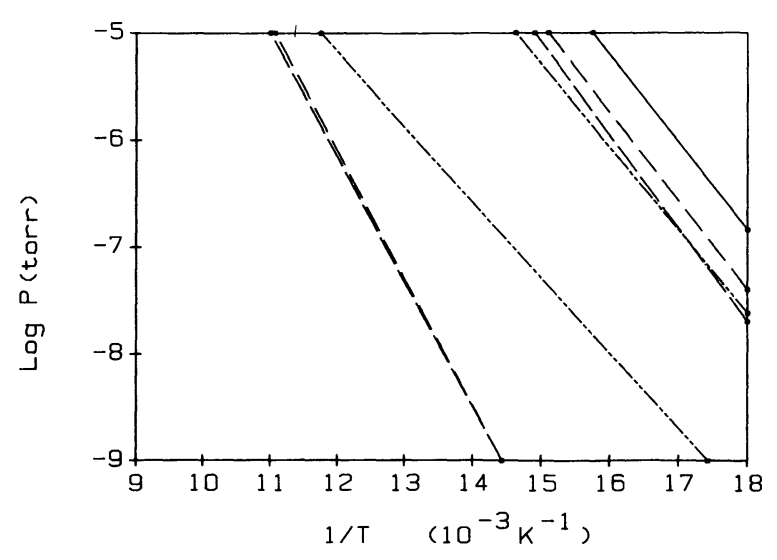

Fig. 1. - Pressures of monolayer and bilayer condensation of adsorbed xenon as a function of temperature. The logarithm of the pressure of coexisting three-dimensional gas, in torr, is plotted as a function of inverse temperature (in $\mathrm{K}^{-1}$ ) for $\mathrm{Xe} / \mathrm{Ag}(111)$ (dot-dash lines, Ref. [1]), $\mathrm{Xe} / \mathrm{Gr}$ (dashed lines, Refs. [4] and [34]), and $\mathrm{Xe} / \mathrm{Pd}(100)$ (dot-dotdash lines, Ref. [35]). The extrapolation of the bulk sublimation data of reference [36] to lower temperatures is also shown (solid line). The ordering of the bilayer condensation is different from the monolayer ordering; see section 2 .

tinuity [4] at the solid bilayer formation for $\mathrm{Xe} / \mathrm{Gr}$. The insensitivity of the second-layer formation to the first-layer adsorption-induced interactions is strikingly shown in the $\mathrm{Xe} / \mathrm{Pd}(100)$ data : for the conditions represented in figure 1 there was [35] no 2D island formation and no sharp monolayer condensation for the $\mathrm{Xe} / \mathrm{Pd}(100)$, but the bilayer condensation is in the same regime as the other two cases.

Data for the bilayer formation of $\mathrm{Ar}$ and $\mathrm{Kr}$ on $\operatorname{Ag}(111)[1]$ and on $\operatorname{Gr}[2,3]$ also only depend weakly on $\varepsilon_{0}$. For example, the low coverage isosteric heat for $\mathrm{Ar} / \mathrm{Gr}$ is $20 \%$ larger than for $\mathrm{Ar} / \mathrm{Ag}(111)$ [29] and the pressure of the coexisting $3 \mathrm{D}$ Ar gas at the monolayer condensation at $45 \mathrm{~K}$ is 50 times larger for $\operatorname{Ag}(111)$ [1] than for Gr [2]. Nevertheless, the estimated 3D gas pressure for the bilayer condensation on $\mathrm{Gr}$ $[37,2]$ is 2 times larger than on $\operatorname{Ag}(111)$ [1]. A calculation of the bilayer condensation pressure using a realistic interaction model for $\mathrm{Ar} / \mathrm{Gr}$ gave very good agreement [9] with pressures extrapolated from Gilquin's data [37]. Gilquin also reported a multilayer isotherm at $64 \mathrm{~K}$ [37] which followed the SingletonHalsey equation [33]. However, calculating the energy coefficient of the Singleton-Halsey equation in terms of the polarization potentials of Ar to bulk Ar and to $\mathrm{Gr}$ gives the same $50 \%$ underestimate by theory already reported for $\mathrm{Kr}$ and $\mathrm{Xe}$ multilayers on $\mathrm{Gr}$ [38]. The modelling neglects thermally activated processes in the multilayer adsorption, which may account for this discrepancy.

The bilayer condensation for ${ }^{4} \mathrm{He} / \mathrm{Gr}$ deviates from the pattern of the classical gases. The ${ }^{4} \mathrm{He} / \mathrm{Gr}$ monolayer is highly compressible, as a result of quantum effects, and $\varepsilon_{0}$ is larger relative to the attractive forces 
in the lateral interactions than for the heavier inert gases. The monolayer compression has been inferred from thermodynamic data $[39,40]$ and has been observed in neutron diffraction experiments [41]. The second layer at bilayer formation is believed to be a 2D quantum liquid $[39,41]$ which evolves to a solid after a further chemical potential increase. There are alternative interpretations [41] of the neutron diffraction data for the bilayer solid as reflecting two mutually incommensurate solid layers of different densities or as reflecting a mutually commensurate bilayer solid with an oblique unit cell. If the bilayer condenses directly into either of these structures, equation (2.7) is violated by $10 \%$ and there is a direct contribution of $\varepsilon_{0}$ in $\mu_{\mathrm{II}}$, as indicated by equation (2.5). We develop the analysis of bilayer condensation here for the case of condensation of second-layer quantum liquid; again the chemical potential at bilayer condensation $\mu_{\mathrm{II}}$ is only indirectly dependent on $\varepsilon_{0}$.

The internal energy and chemical potential of the He monolayer are given by equations (2.1) and (2.3), where $\varepsilon_{\rho_{1}}$ is now a quantum 2D equation of state of compressed $\mathrm{He}$ which, for approximations, may be taken from model calculations [42-44] or from the extensive thermodynamic data for monolayer [40] $\mathrm{He} / \mathrm{Gr}$.

For the bilayer we use a different formulation than equation (2.2), to make contact with model calculations [43] and to reflect more directly the terms giving rise to the spreading pressure. Let the total number of atoms in the bilayer $N_{2}$ be divided into $N_{21}$ firstlayer and $N_{22}$ second-layer atoms, with areas per atom $\alpha_{1}$ and $\alpha_{2}$, respectively [45]. The number and area constraints are :

$$
\begin{aligned}
N_{2} & =N_{21}+N_{22} \\
N_{21} \alpha_{1} & =N_{22} \alpha_{2}=N_{2} a_{2} .
\end{aligned}
$$

We take as an approximation for the bilayer energy

$$
\begin{aligned}
u_{2}=\left(N_{21} / N_{2}\right) & {\left[\varepsilon_{0}+\varepsilon_{\ell 1}\left(\alpha_{1}\right)\right]+} \\
+ & \left(N_{22} / N_{2}\right)\left[v_{2}\left(\alpha_{1}\right)+v_{22}\left(\alpha_{2}\right)\right] .
\end{aligned}
$$

Here $\varepsilon_{0}$ is the binding energy of first-layer adatoms to the substrate and $v_{2}\left(\alpha_{1}\right)$ is the binding energy of a single second-layer atom to the combined substratefirst-layer system [43]. The lateral energy terms are $\varepsilon_{\ell 1}\left(\alpha_{1}\right)$ for the first-layer solid [40,42] and $v_{22}\left(\alpha_{2}\right)$ for the (second-layer) 2D quantum liquid [44].

The condition for mass transfer equilibrium between the first and second layers of the bilayer is

$$
\begin{aligned}
\mu_{2}= & v_{2}\left(\alpha_{1}\right)+v_{22}\left(\alpha_{2}\right)-\alpha_{2} \mathrm{~d} v_{22} / \mathrm{d} \alpha_{2} \\
= & \varepsilon_{0}+\varepsilon_{{ }_{1}}\left(\alpha_{1}\right)-\alpha_{1} \mathrm{~d} \varepsilon_{\ell 1} / \mathrm{d} \alpha_{1}- \\
& -\left(N_{22} \alpha_{1} / N_{21}\right) \mathrm{d} v_{2} / \mathrm{d} \alpha_{1} .
\end{aligned}
$$

The conditions of mechanical and mass transfer equilibrium between the monolayer and bilayer are :

$\phi_{1}=-\left[\mathrm{d} \varepsilon_{p 1} / \mathrm{d} \alpha_{1}+\mathrm{d} v_{22} / \mathrm{d} \alpha_{2}+\left(N_{22} / N_{21}\right) \mathrm{d} v_{2} / \mathrm{d} \alpha_{1}\right]$

$\mu_{1}=\mu_{2}=\mu_{\mathrm{II}}$.
There is evidence [39] that the initial bilayer of ${ }^{4} \mathrm{He} / \mathrm{Gr}$ has a low-density quantum liquid in the second layer $\left(N_{22} / N_{21} \lesssim 0.3\right)$. Then equations (2.11) and (2.12) have an approximate solution with

$$
\begin{aligned}
& \mathrm{d} v_{22} / \mathrm{d} \alpha_{2} \simeq 0 \\
& a_{1} \simeq \alpha_{1}+\left\{\left(N_{22} / N_{21}\right)\left(\mathrm{d} v_{2} / \mathrm{d} \alpha_{1}\right) / \mathrm{d}^{2} \varepsilon_{\ell 1} / \mathrm{d} \alpha_{1}^{2}\right\} \\
& \mu_{\mathrm{II}} \simeq v_{2}\left(\alpha_{1}\right)+v_{22}\left(\alpha_{2}\right) .
\end{aligned}
$$

Thus $\mu_{\mathrm{II}}$ is again only weakly dependent on $\varepsilon_{0}$, through the dependence of the second-layer holding energy $v_{2}$ on the first-layer density. However, the extent of the compression of the monolayer density depends on the difference $\mu_{\mathrm{II}}-\mu_{\mathrm{I}}$.

Using the $2 \mathrm{D}{ }^{4} \mathrm{He}$ equation of state $[40,42]$ and the $\varepsilon_{0}$ for $\mathrm{He} / \mathrm{Au}(110)$ [15] in place of the value for ${ }^{4} \mathrm{He} / \mathrm{Gr}$ leads to an estimate that the monolayer completion density will be $20 \%$ smaller on $\mathrm{Au}(110)$ than on Gr. The 3D gas pressure for monolayer ${ }^{4} \mathrm{He} / \mathrm{Au}(110)$ is larger than $10^{-5}$ torr above $3.5 \mathrm{~K}$, so that ${ }^{4} \mathrm{He}$ monolayer adsorption on $\mathrm{Au}$ is experimentally accessible [46] near $4 \mathrm{~K}$. There are already large thermal excitation effects in the He layers at such temperatures.

We have discussed the monolayer-to-bilayer transition primarily in terms of solid monolayers, but for very weak holding potentials the monolayer solid of He may not occur, as already noted by Richards [47] for the case of ${ }^{3} \mathrm{He}$ adsorbed on $\mathrm{H}_{2}$. In $3 \mathrm{D}$ the chemical potential changes [48] at zero temperature from liquefaction to solidification are $7.6 \mathrm{~K}$ for ${ }^{4} \mathrm{He}$ and $12.2 \mathrm{~K}$ for ${ }^{3} \mathrm{He}$. For ${ }^{3} \mathrm{He}$ at $2 \mathrm{~K}$ the required chemical potential increase is $24.4 \mathrm{~K}$. Model calculations [44] for the solidification of ${ }^{4} \mathrm{He}$ at $0 \mathrm{~K}$ in mathematical 2D can be used to make an estimate of $7 \mathrm{~K}$ for the zero temperature chemical potential increase from $2 \mathrm{D}$ liquefaction to solidification; the increase for $2 \mathrm{D}^{3} \mathrm{He}$ would be larger and might surpass the $10 \mathrm{~K}$ difference between the $\varepsilon_{0}$ of ${ }^{3} \mathrm{He} / \mathrm{H}_{2}[31,32]$ and the chemical potential $\mu_{0} \simeq-2.5 \mathrm{~K}$ of $3 \mathrm{D}$ liquid ${ }^{3} \mathrm{He}$ at $0 \mathrm{~K}$. There have been some calculations [32] of the holding potential and bound states of ${ }^{3} \mathrm{He}$ on $\mathrm{H}_{2}$. A further investigation might include consideration of possible reorganization [49] of the bilayer $\mathrm{H}_{2}$ film, of the effect of anisotropy [50] of the $\mathrm{H}_{2}$ vibrations on the corrugation of the He holding potential, and of the effect of the coupling of the motions of the $\mathrm{He}$ and $\mathrm{H}_{2}$ on the equation of state of the adsorbed $\mathrm{He}$. The bilayer transition for close-packed triangular monolayer and bilayer lattices has been treated with quasiharmonic lattice dynamics [8] for $D_{2}$ parameters [51]; some information on these questions can be obtained with the phonon spectra calculated in that work.

\section{A limit of stability for $\mathrm{Xe} / \mathrm{Cu}(\mathbf{1 1 0})$.}

At monolayer condensation, $\mathrm{Xe}$ forms a $\mathrm{C}(2 \times 2)$ lattice on $\mathrm{Cu}(110)[20,21]$. Under compression, the 
Xe monolayer becomes a uniaxially registered centred rectangular lattice : the Xe spacing along the [1히 axis of the $\mathrm{Cu}$ surface decreases while the spacing between $\mathrm{Xe}$ rows in the [001] direction remains constant. In the LEED experiments of Glachant et al. [21] near $70 \mathrm{~K}$, a chemical potential increase of $800 \mathrm{~K}$ produced a $12 \%$ decrease in the Xe spacing along the [110] axis from the $5.1 \AA$ value at monolayer condensation. There was no evidence for bilayer or bulk formation [21]; however, Mason and Williams [22], with dosing experiments and analysis by $\mathrm{He}$ atom scattering, observed an expanded Xe structure at nominal coverages larger than the monolayer coverage.

Previously [28] the LEED data for $\mathrm{Xe} / \mathrm{Cu}(110)$ $[20,21]$ were used to make estimates of the adatomadatom and adatom-substrate interactions. Estimates were obtained for the leading Fourier amplitudes $2 V_{1}$ and $2 V_{2}$ of the holding potential along the [001] and $[1 \overline{1} 0]$ axes. The adatom-adatom interactions were represented by Lennard-Jones 12-6 pair potentials of depth $\varepsilon$ and separation $r_{0}$ at the potential minimum. Values of $\varepsilon=230 \mathrm{~K}$ and $r_{0}$ in the range 4.5 to $4.55 \AA$ led to results for the monolayer compressibility which were in fair agreement with the LEED data [21]. Those calculations [28] did not depend on the value for the monolayer heat of adsorption $\left(q_{1}\right)$.

In this section we formulate a zero temperature theory of the limit of stability of the $\mathrm{Xe} / \mathrm{Cu}(110)$ monolayer against bilayer formation. We assume the monolayer remains in uniaxial registry, with zero misfit $M_{1}$ relative to the [001] row spacing of the $\mathrm{Cu}$, up to the bilayer (or bulk) condensation. In the previous calculations [28], for values of $V_{1}$ required in order that the monolayer condense with $M_{1}=0$, the monolayer remained in a state of zero $M_{1}$ for large chemical potential increases. We assume also that the bilayer is in uniaxial $\left(M_{1}=0\right)$ registry with the substrate; this may be too strong a constraint and might lead to a fictitious stability of the compressed monolayer relative to bilayer formation. However, with the present experimental information on the first-layer holding potential, the compressed monolayer is calculated to be unstable with respect to the bilayer at lattice misfit and chemical potential increase corresponding to an experimentally stable monolayer [21]; relaxing the constraint on the bilayer structure in the calculation could only increase the monolayer instability. The calculations provide another example satisfying equation (2.8) : the chemical potential at the bilayer condensation is insensitive to $\varepsilon_{0}$.

We extend the original zero temperature theory of bilayer formation for triangular lattices [7] by treating centred rectangular lattices and by including effects

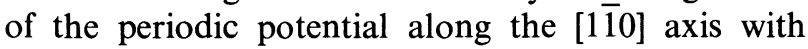
perturbation theory [28]. The energies $\varepsilon_{p i}$ of equations (2.1) and (2.2) are replaced by potential energies of the modulated centred rectangular lattices. If $l$ denotes the nearest-neighbour spacing in the $\mathrm{Cu}(2.55 \AA)$ and $M_{2}$ denotes the misfit of the Xe spacing along the [1하] direction relative to the $\mathrm{C}(2 \times 2)$ lattice, the areas $a_{i}$ are

$$
\begin{aligned}
& a_{1}=l^{2} 2 \sqrt{2}\left(1+M_{2}\right) \\
& a_{2}=l^{2} \sqrt{2}\left(1+M_{2}\right) .
\end{aligned}
$$

In a Cartesian coordinate system with $\hat{\mathbf{x}}$ along the [1-10] direction and $\mathbf{y}$ along the [001] direction, the basis vectors for the centred rectangular lattices are

$$
\begin{aligned}
& \mathbf{b}_{1}=\left(1+M_{2}\right) l \hat{\mathbf{x}}+l \sqrt{2} \hat{\mathbf{y}} \\
& \mathbf{b}_{2}=-\left(1+M_{2}\right) l \hat{\mathbf{x}}+l \sqrt{2} \hat{\mathbf{y}} .
\end{aligned}
$$

Apart a choice of the origin of coordinates, the centred rectangular lattice consists of points

$$
\mathbf{R}_{j}=N_{1} \mathbf{b}_{1}+N_{2} \mathbf{b}_{2}, \quad N_{1}, N_{2}=\text { integers } .
$$

We assume the modulated positions of the first- and second-layer atoms can be represented by

$$
\begin{aligned}
& \mathbf{r}_{j 1}=\mathbf{R}_{j}+u\left(\mathbf{R}_{j}\right) \hat{\mathbf{x}} \\
& \mathbf{r}_{j 2}=\mathbf{R}_{j}+y \hat{\mathbf{y}}+v\left(\mathbf{R}_{j}\right) \hat{\mathbf{x}}+z_{12} \hat{\mathbf{z}} .
\end{aligned}
$$

The first of equations (3.4) expresses the constraint that the first-layer atoms are in uniaxial registry with the $\mathrm{Cu}(110)$. The second of equations (3.4) includes a shift of the second-layer origin relative to the firstlayer and a modulation of the second-layer positions along the $x$-axis only.

The substrate potential for the first-layer adatoms is represented by

$$
\begin{aligned}
V\left(\mathbf{r}_{j 1}\right) & =\varepsilon_{0}-2 V_{2} \cos \left(\frac{2 \pi}{l} \hat{\mathbf{x}} \cdot \mathbf{r}_{j}\right) \\
& =\varepsilon_{0}-2 V_{2} \cos \left(\mathbf{q} \cdot \mathbf{R}_{j}+\frac{2 \pi}{l} u\left(\mathbf{R}_{u}\right)\right)
\end{aligned}
$$

where the misfit wave vector is

$$
\mathbf{q}=\frac{2 \pi M_{2}}{l\left(1+M_{2}\right)} \hat{\mathbf{x}}
$$

The separation of $\varepsilon_{0}$ into a laterally averaged energy and the corrugation amplitude $2 V_{1}$ is not required here [28]. We denote the average first-layer corrugation potential energy for $N_{1}$ first-layer atoms by

$$
\bar{v}_{\mathrm{cp}}=-\left(2 V_{2} / N_{1}\right) \sum_{j=1}^{N_{1}} \cos \left[\mathbf{q} \cdot \mathbf{R}_{j}+\frac{2 \pi}{l} u\left(\mathbf{R}_{j}\right)\right] \text {. }
$$

The second-layer adatoms interact with the substrate by the polarization potential

$$
\varepsilon_{\mathrm{II}}=-C_{3} /\left(z_{12}+z_{0}\right)^{3},
$$

where $z_{12}$ is the interplanar spacing of the bilayer, $C_{3}$ is the strength coefficient $\left(3.93 \times 10^{4} \mathrm{~K}^{3}\right.$ for $\mathrm{Xe} / \mathrm{Cu}[30])$ and $z_{0}$ is the distance from the first-layer to an effective electrodynamical image plane. In the absence of information specific to the $\mathrm{Xe} / \mathrm{Cu}(110)$ 
system, we take a value $z_{0}=2 \AA$ used for $\mathrm{Xe} / \mathrm{Ag}(111)$ models $[7-9] ; 10 \%$ uncertainty in $z_{0}$ leads to $10 \%$ uncertainty in $\varepsilon_{\mathrm{II}}$ for the $\mathrm{Xe} / \mathrm{Cu}(110)$.

For the lateral interactions among the adatoms we use the Lennard-Jones 12-6 potential

$$
\phi(r)=\varepsilon\left\{\left(r_{0} / r\right)^{12}-2\left(r_{0} / r\right)^{6}\right\},
$$

with $\varepsilon=230 \mathrm{~K}$ and two choices of $r_{0}, 4.5$ and $4.55 \AA$. Sums of $\phi(r)$ for atoms both in the first-layer, both in the second-layer and one in each layer are defined by

$$
\begin{aligned}
& \Phi_{11}=\frac{1}{2} \sum_{j \neq 0} \phi_{11}\left(\left|\mathbf{r}_{j 1}-\mathbf{r}_{01}\right|\right) \\
& \Phi_{22}=\frac{1}{2} \sum_{j \neq 0} \phi_{22}\left(\left|\mathbf{r}_{j 2}-\mathbf{r}_{02}\right|\right) \\
& \Phi_{12}=\sum_{j} \phi_{12}\left(\left|\mathbf{r}_{j 1}-\mathbf{r}_{02}\right|\right) .
\end{aligned}
$$

This notation permits a separation of the effects of substrate-mediated interactions for first- and secondlayer atoms; here, however, we use the same potential function $\phi$, equation (3.9), throughout equations (3.10).

The internal energies for the monolayer and bilayer are written in terms of these functions as

$$
\begin{aligned}
& u_{1}=\varepsilon_{0}+\bar{v}_{\mathrm{cp}}+\Phi_{11} \\
& u_{2}=\left(\varepsilon_{0}+\bar{v}_{\mathrm{cp}}+\varepsilon_{\mathrm{II}}+\Phi_{11}+\Phi_{22}+\Phi_{12}\right) / 2 .
\end{aligned}
$$

The energy $u_{1}$ is evaluated as a function of $M_{2}\left(a_{1}\right)$, which requires determining the optimum modulation $u\left(\mathbf{R}_{j}\right)$ to minimize the energy at each value of $M_{2}$. For the energy $u_{2}$ the two structural parameters $y$ and $z_{12}$ should be optimized, as well as the modulation functions $u\left(\mathbf{R}_{j}\right)$ and $v\left(\mathbf{R}_{j}\right)$. In our calculations, we use a perturbation approach and optimize the parameters $y$ and $z_{12}$ as a function of $M_{2}\left(a_{2}\right)$ for the uniform bilayer lattice, $u\left(\mathbf{R}_{j}\right)=v\left(\mathbf{R}_{j}\right)=0$, as a first approximation. The minimization with respect to $y$ is a separate step, not required in previous calculations, which arises here because the second-layer atoms for the centred rectangular bilayer lattice are not in exactly three-fold sites. We then complete the minimization of $u_{1}\left(M_{2}\right)$ and $u_{2}\left(M_{2}\right)$ using a perturbation-variation treatment for the dependences on the functions $u\left(\mathbf{R}_{j}\right)$ and $v\left(\mathbf{R}_{j}\right)$.

We take as trial functions for the modulations of the first- and second-layer adatoms

$$
\begin{aligned}
u_{\alpha}\left(\mathbf{R}_{j}\right) & =A_{\alpha} \sin \left(\mathbf{q} \cdot \mathbf{R}_{j}\right) \\
v\left(\mathbf{R}_{j}\right) & =B \sin \left(\mathbf{q} \cdot \mathbf{R}_{j}+\delta\right)
\end{aligned}
$$

and expand the energies $u_{1}$ and $u_{2}$ in powers of $A_{\alpha}$ and $B$; the amplitude $A_{\alpha}$ differs for the monolayer and bilayer. The energy $\bar{v}_{\mathrm{cp}}$ is carried to the first power of $A_{\alpha}$. The energies $\Phi_{i j}$ are carried to second order in $A_{\alpha}$ and $B$; in those expansions the coefficients of the linear terms vanish and the coefficients of the quadratic terms are similar to the harmonic elastic coefficients used in the previous work [28] for $\mathrm{Xe} / \mathrm{Cu}(110)$. Although we include a phase variable $\delta$ in equation (3.12), the minimum energy $u_{2}\left(M_{2}\right)$ occurs for $\delta$ equal to zero modulo $\pi$. Minimization with respect to $A_{\alpha}$ and $B$ requires only the minimization of a quadratic form.

Within this perturbation theory, we also allowed for a $y$-component to the modulation of the secondlayer positions $r_{j 2}$ in equation (3.4). However, with a modulation function of the form of $v\left(\mathbf{R}_{j}\right)$ in equation (3.12), we found the amplitude of the $y$-modulation to vanish in the minimum energy configuration.

We show the results of such calculations for $\mathrm{Xe} / \mathrm{Cu}(110)$ in figure 2; parameters derived from the calculations are summarized in table I. The chemical potential as a function of spreading pressure is obtained with equation (2.3) using the functions $u_{i}\left(M_{2}\right)$ which have been minimized with respect to $y$, $z_{12}, A_{\alpha}$, and $B$. The misfits at points along the $\mu-\phi$ curves are indicated in the figure. The effect in the results of allowing for the modulations $u\left(\mathbf{R}_{j}\right)$ and $v\left(\mathbf{R}_{j}\right)$, is rather small, as indicated by the dashed symbols in figure 2 showing the results of calculations for the nonmodulated uniform monolayer and bilayer centred

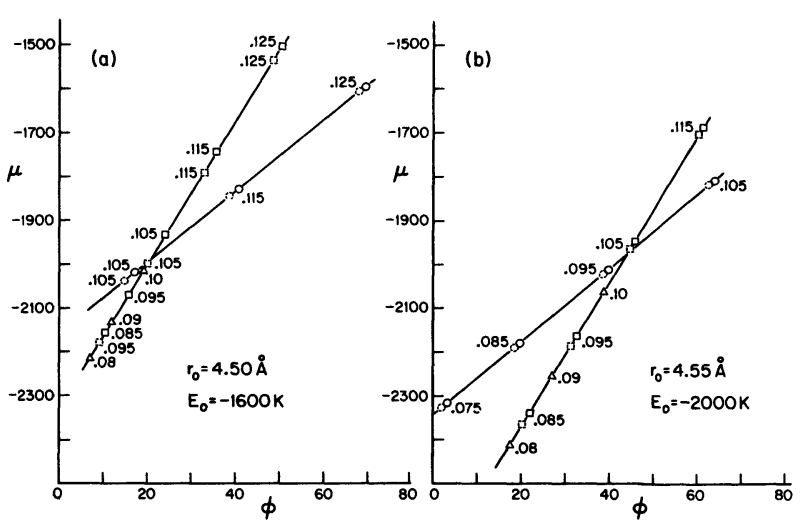

Fig. 2. - Zero temperature chemical potential $\mu$ (in $\mathrm{K}$ ), as a function of spreading pressure $\phi$ (in $\mathrm{K} / \AA^{2}$ ) for uniaxially registered monolayers and bilayers of $\mathrm{Xe}$ on $\mathrm{Cu}(110)$. The circles (squares) denote the results of perturbation calculations for centred rectangular bilayers (monolayers) at the indicated negative misfits $\left(-M_{2}\right)$; see section 3 for a description of the perturbation-variation formalism. The dashed symbols denote results of calculations for uniform nonmodulated lattices. The triangles are results for the monolayer taken from calculations including higher multiples of the misfit wave vector, reference [28]. The smooth lines are interpolations drawn through the points to help locate the monolayer-bilayer equilibrium. The parameters of the model are $\varepsilon_{0}$, the average first-layer holding potential in the [1히] troughs; $2 V_{2}$, the corrugation amplitude along the trough ; $\varepsilon=230 \mathrm{~K}$ and $r_{0}$ the energy and length scales of the LennardJones pair potential, equation (3.9). The results of changing the $\varepsilon_{0}$ value by $\Delta \varepsilon$ are obtained by shifting the monolayer $\mu$ by $\Delta \varepsilon$ and the bilayer $\mu$ by $\Delta \varepsilon / 2$. a) $r_{0}=4.5 \AA, \varepsilon_{0}=$ $-1600 \mathrm{~K}$; b) $r_{0}=4.55 \AA, \varepsilon_{0}=-2000 \mathrm{~K}$. 
Table I. - Monolayer-bilayer coexistence for models of uniaxially registered $\mathrm{Xe} / \mathrm{Cu}(110)$.

\begin{tabular}{|c|c|c|c|c|}
\hline$r_{0}(\AA)\left(^{a}\right)$ & & & & \\
\hline$\varepsilon_{0}(\mathrm{~K})\left(^{\mathrm{b}}\right)$ & -1600 & -2000 & -1600 & -2000 \\
\hline$v_{2}\left({ }^{c}\right)$ & 0.19 & 0.19 & 0.09 & 0.09 \\
\hline$\mu_{\mathrm{I}}(\mathrm{K})\left(^{\mathrm{d}}\right)$ & -2342 & -2742 & -2323 & -2723 \\
\hline$\mu_{\mathrm{II}}(\mathrm{K})\left({ }^{\mathrm{e}}\right)$ & -1997 & -1996 & -1979 & -1978 \\
\hline$\mu_{\mathrm{II}}-\mu_{\mathrm{I}}(\mathrm{K})$ & 345 & 746 & 344 & 745 \\
\hline$\phi\left(\mathrm{K} / \AA^{2}\right)\left({ }^{f}\right)$ & 20.3 & 44.8 & 19.8 & 43.9 \\
\hline$M_{2_{1}}(\mathrm{~g})$ & 0.101 & 0.122 & 0.082 & 0.104 \\
\hline$M_{2_{2}}(\mathrm{~h})$ & 0.106 & 0.117 & 0.085 & 0.097 \\
\hline
\end{tabular}

(a) Separation at the $\mathrm{LJ}(12-6)$ potential minimum, equation (3.9). The energy scale is $\varepsilon=230 \mathrm{~K}$ and the $\mathrm{Cu}$ spacing is $l=2.55 \AA$.

(b) Single-adatom holding potential in first-layer trough, see equation (3.5).

(c) Reduced corrugation amplitude is [1 $\overline{1} 0]$ direction, $v_{2}=2 V_{2} / \varepsilon$, equation (3.5), from reference [28].

(d) Chemical potential at monolayer condensation in $\mathrm{C}(2 \times 2)$ lattice.

( ${ }^{\mathrm{e}}$ Chemical potential at bilayer condensation; for this model bulk condensation occurs at $\mu_{0}=-1980 \mathrm{~K}$.

(f) Spreading pressure at the bilayer condensation.

(g) Misfit $M_{2_{1}}$ of the monolayer lattice at bilayer condensation.

(b) Misfit $M_{2_{2}}$ of the bilayer lattice at condensation.

rectangular lattices. The adequacy of the perturbation treatment for these values of $M_{2}$ and $V_{2}$ is tested by comparing the monolayer results with data from a more complete treatment of the monolayer distortions where phenomena such as misfit dislocations were treated [28]. Results of that earlier work are included in figure 2 and agree well with the $\mu(\phi)$ function derived from the perturbation theory.

As is apparent from figure 2, the chemical potential, spreading pressure, and misfits at the monolayer-tobilayer transition change little whether or not the modulations of the adlayers are included in the calculations. The changes in the equilibrium misfits from the calculation with modulations to the calculation for the unmodulated lattices are about -0.002 in $M_{2_{1}}$ and -0.001 in $M_{22}$. These amount to changes in the Xe spacing along the [1힐 trough of less than $0.02 \AA$, which are smaller than the uncertainties in the lengths derived from the LEED experiments [20, 21].

Changing the value $\varepsilon_{0}$ of the first-layer holding potential by $400 \mathrm{~K}$ changes $\mu_{\text {II }}$ by less than $5 \mathrm{~K}$, which agrees with the discussion of section 2 . However, the spreading pressure $\phi$ and the misfits $M_{2_{1}}$ and $M_{2_{2}}$ of the coexisting monolayer and bilayer do depend noticeably on $\varepsilon_{0}$, as shown in table I. The results in table I are obtained from the equation of state with the modulation treated as a perturbation. The discontinuity in the $\mathrm{Xe}$ nearest-neighbour spacing along the [11 0$]$ direction at the bilayer condensation is about $0.5 \%$ for the various cases treated in these calculations.

There are two more ways in which the calculated monolayer-to-bilayer transition can be compared to the data for $\mathrm{Xe} / \mathrm{Cu}(110)[20-22]$ : the extent of mono- layer compression without bilayer formation and the occurrence of a bilayer before bulk condensation.

The calculated $\mu_{\text {II }}$ values are exceedingly close to the chemical potential $\mu_{0}$ for bulk condensation [52] with the LJ (12-6) potential model we have used, $\mu_{0}=-1980 \mathrm{~K}$. The two $r_{0}$ cases give $\mu_{\text {II }}$ values within $20 \mathrm{~K}$ of $\mu_{0}$, and on opposite sides of it, so that the appearance of a bilayer before bulk condensation is highly sensitive to the compressibility of the layer. It also depends on the value taken for $z_{0}$ in equation (3.8). The data shown in figure 1 correspond to $\mu_{0}-\mu_{\mathrm{II}}$ differences at $60 \mathrm{~K}$ of $75 \mathrm{~K}$ for $\mathrm{Xe} / \mathrm{Gr}$ and $110 \mathrm{~K}$ for $\mathrm{Xe} / \mathrm{Ag}(111)$. The fact that $\mu_{\mathrm{II}}$ is so close to $\mu_{0}$ for the $\mathrm{Xe} / \mathrm{Cu}(110)$ model is in accord with the idea that the constraint imposed by uniaxial registry may limit the growth to a single compressed monolayer before bulk formation. However, the $\mathrm{Xe} / \mathrm{Cu}(110)$ interaction model is still rather primitive, with no explicit inclusion of adsorption-induced or substratemediated interactions [30]. These calculations show the combined effect of two structural considerations $[11,13]$, compression and registry, in limiting bilayer formation before bulk condensation. The growth mode for the physical system $\mathrm{Xe} / \mathrm{Cu}(110)$ remains an open question.

The difference $\mu_{\mathrm{II}}-\mu_{\mathrm{I}}$ is linked to the amount of monolayer compression which occurs before bilayer formation. Initially we used the experimental $q_{1}$ value [21] for the latent heat of condensation in the $\mathrm{C}(2 \times 2)$ lattice to determine a first-layer holding potential $\varepsilon_{0}=-1600 \mathrm{~K}$. However, as shown by the entries in table I, there would then be only a chemical potential increase of $350 \mathrm{~K}$ permitted before bilayer (or 
bulk) condensation. Near $70 \mathrm{~K}$ Glachant et al. [21] were able to increase the chemical potential by about $800 \mathrm{~K}$ without condensation beyond the monolayer; thermal effects alone do not seem large enough to account for the difference. Also, they observed monolayer misfits $M_{2}$ more negative than -0.11 , which would not be available to the monolayer in our model calculations with $\varepsilon_{0}=-1600 \mathrm{~K}$. For these reasons we repeated the calculations with $\varepsilon_{0}=$ $-2000 \mathrm{~K}$, which corresponds to $q_{1} \simeq-2700 \mathrm{~K}$ and is a holding potential more in the range found for Xe adsorbed on $\mathrm{Ag}(111)$ and on basal plane graphite [29]. Then, as shown in table I, the chemical potential increase from the $\mathrm{C}(2 \times 2)$ condensation to bilayer formation is about $750 \mathrm{~K}$ and the monolayer limit of compression is more in keeping with the LEED data [21]. As in the previous work [28], using the value $r_{0}=4.5 \AA$ reproduces the monolayer data a little better than calculating with the value $4.55 \AA$.

The Glachant et al. data [21, 53] become consistent with the adjusted $\varepsilon_{0}$ value if the two lowest temperature data points are dropped from the determination of $q_{1}$. The monolayer latent heat $\left(q_{1}\right)$ is derived from the pressure-temperature data for the $\mathrm{C}(2 \times 2)$ condensation and deleting the lowest temperature points [53] from the slope determination leads to an increase of more than $10 \%$ in the $q_{1}$ value. The actual magnitudes of the transition temperatures and pressures in the experiments [21] were close to the $\mathrm{Xe} / \operatorname{Ag}(111)$ values [1], but including the lowest temperature points led to an anomalously small $q_{1}$ compared to the $\mathrm{Xe} / \mathrm{Cu}(100)$ value [54] and to an anomalous entropy of adsorption [53] for $\mathrm{Xe} / \mathrm{Cu}(110)$.

There were recognized to be slow adsorption kinetics $[21,53]$ at the lowest experimental temperatures, so dropping those points from the $q_{1}$ determination has some basis. We must note that our calculations are for zero temperature and the experiments near $70 \mathrm{~K}$ involve adlayers with thermal excitation. The change we propose for $q_{1}$ makes the systematic trends in the comparisons with $\mathrm{Xe} / \mathrm{Cu}(100)$ [54] and $\mathrm{Xe} / \mathrm{Ag}(111)$ [1] smoother.

\section{Concluding remarks.}

We have discussed the similarity of the value of the chemical potential at bilayer condensation $\mu_{\mathrm{II}}$ for a given near-classical adsorbate on several substrates and have related it to the absence of (or small) lattice constant discontinuities in the adsorbate at the monolayer-to-bilayer transition. The analysis shows how the weakening of direct dependences of $\mu_{\text {II }}$ on the binding energy $\varepsilon_{0}$ of first-layer adatoms to the substrate and on adsorption-induced interactions among first-layer adatoms arises. We extended the discussion to the case of adsorbed helium for which the values of $\varepsilon_{0}$ derived from atom-surface scattering experiments are very different for noble metals and for graphite. The absence of a direct dependence of $\mu_{\text {II }}$ on $\varepsilon_{0}$ occurs again, but the monolayer ${ }^{4} \mathrm{He}$ density at the limit of compression may be $20 \%$ less on $\mathrm{Au}$ than on $\mathrm{Gr}$.

We presented a model calculation for the limit of monolayer compression of uniaxially registered $\mathrm{Xe} / \mathrm{Cu}(110)$, where there may be an absence of bilayer formation before bulk condensation because of a combination of the structural effects of registry and compression. In order to account for the observed compression, we adjusted the value for $\varepsilon_{0}$ in a way which makes the $\mathrm{Xe} / \mathrm{Cu}(110)$ holding potential fall more smoothly onto trends for $\mathrm{Xe} / \mathrm{Cu}(100)$ and $\mathrm{Xe} / \mathrm{Ag}(111)$.

Much work remains to be done in following the evolution of uniaxially registered monolayers to higher coverages and to relate the properties to other layer-growth observations.

\section{Acknowledgments.}

During this work one of us (LWB) was appointed as Professeur Associé at the Université d'Aix-Marseille II, Centre de Luminy and was also supported in part by the U.S. National Science Foundation Grant DMR-8214518. This material is based in part upon work supported by the North Atlantic Treaty Organization under a grant awarded in 1984 to JK. We thank Professeur M. Bienfait for his hospitality.

References

[1] Unguris, J., BruCh, L. W., Moog, E. R. and Webb, M. B., Surface Sci. 87 (1979) 415; 109 (1981) 522.

[2] Shaw, C. G. and Fain, S. C., Jr., Surface Sci. 91 (1980) L1; 83 (1979) 1.

[3] Fain, S. C., Jr. and Chinn, M. D., J. Physique 38 (1977) C4-99.

[4] Schabes-Retchiman, P. S. and Venables, J. A., Surface Sci. 105 (1981) 536.

[5] Tiby, C., Wiechert, H. and Lauter, H. J., Surface Sci. 119 (1982) 21.

[6] Calisti, S., Suzanne, J. and Venables, J. A., Surface Sci. 115 (1982) 455.
[7] Bruch, L. W., Unguris, J. and WebB, M. B., Surface Sci. 87 (1979) 437.

[8] Bruch, L. W. and WeI, M. S., Surface Sci. 100 (1980) 481.

[9] Wei, M. S. and BruCh, L. W., J. Chem. Phys. 75 (1981) 4130.

[10] Bruch, L. W., Phillips, J. M. and Ni, X.-Z., Surface Sci. 136 (1984) 361.

[11] Muirhead, R. J., Dash, J. G. and Krim, J., Phys. Rev. B 29 (1984) 5074 ;

Pandit, R., Schick, M. and Wortis, M., Phys. Rev. B 26 (1982) 5112. 
[12] Seguin, J. L., Suzanne, J., Bienfait, M., Dash, J. G. and Venables, J. A., Phys. Rev. Lett. 51 (1983) 122 ;

Venables, J. A., Seguin, J. L., Suzanne, J. and BIENFAIT, M., Surface Sci. 145 (1984) 345.

[13] Venables, J. A., Spiller, G. D. T. and Hanbücken, M., Rept. Progr. Phys. 47 (1984) 399 and references contained therein.

[14] Cole, M. W., Frankl, D. R. and Goodstein, D. L., Rev. Mod. Phys. 53 (1981) 199 and references contained therein.

[15] Rieder, K. H., Engel, T. and Garcia, N., Proc. ECOSS-3, Vol. II, p. 861 (Cannes, 1980) [Supplément à Le Vide, les Couches Minces, no 201].

[16] Luntz, A., Mattera, L., Rocca, M., Tommasini, F. and Valbusa, U., Surface Sci. 120 (1982) L447.

[17] Perreau, J. and Lapujoulade, J., Surface Sci. 122 (1982) 341.

[18] Liebsch, A. and Harris, J., Surface Sci. 130 (1983) L349;

Yu, C. F., Whaley, K. B., Hogg, C. S. and Sibener, S. J., Phys. Rev. Lett. 512210 (1983);

Yu, C. F., Whaley, K. B., Hogg, C. S. and Sibener, S. J., submitted to J. Chem. Phys.

[19] Mattera, L., Rosatelli, F., Salvo, C., Tommasini, F., Valbusa, U. and Vidali, G., Surface Sci. 93 (1980) 515.

[20] Chesters, M. A., Hussain, M. and Pritchard, J., Surface Sci. 35 (1973) 161.

[21] Glachant, A., Jaubert, M., Bienfait, M. and BoAto, G., Surface Sci. 115 (1981) 219 ;

Glachant, A., Bienfait, M. and Jaubert, M. (to be published).

[22] Mason, B. F. and Williams, B. R., Surface Sci. 130 (1983) $295 ; 139$ (1984) 173.

[23] Coulomb, J. P., Bienfait, M. and Thorel, P., J. Physique 42 (1981) 293 and references contained therein.

[24] Vora, P., Sinha, S. K. and Crawford, R. K., Phys. Rev. Lett. 43 (1979) 704.

[25] Baer, D. R., FraAss, B. A., Riehl, D. H. and SimMONS, R. O., J. Chem. Phys. 68 (1978) 141.

[26] Goodstein, D. L., Hamilton, J. J., LyseK, M. J. and Vidali, G., Surface Sci. (to be published).

[27] Nielsen, M., McTague, J. P. and Ellenson, W., J. Physique Colloq. 38 (1977) C4-10.

[28] BruCH, L. W., Surface Sci. (in press).

[29] Unguris, J., Bruch, L. W., WebB, M. B. and PhilLIPS, J. M., Surface Sci. 114 (1982) 219.

[30] Bruch, L. W., Surface Sci. 125 (1983) 194 and references contained therein.

[31] Lefevre-Seguin, V., Thèse d'état, Paris (1984) (unpublished).

[32] Pierre, L., Guignes, H. and Lhuillier, C. (to be published).

[33] Singleton, J. H. and Halsey, G. D., Jr., Can. J. Chem. 33 (1955) 184.

[34] Suzanne, J., Coulomb, J. P. and Bienfait, M., Surface Sci. 47 (1975) 204.
[35] Moog, E. R., Ph. D. thesis, University of WisconsinMadison (1984), (unpublished);

Moog, E. R. and WebB, M. B., Surface Sci. (in press).

[36] Tessier, C., Terlain, A. and Larher, Y., Physica 113A (1982) 286.

[37] Gilquin, B., Thèse, Nancy (1979) (unpublished); LARHER, Y., Surface Sci. 134 (1983) 469.

[38] Bruch, L. W. and Watanabe, H., Surface Sci. 65 (1977) 619.

[39] Polanco, S. E. and Bretz, M., Phys. Rev. B 17 (1978) 151.

[40] Elgin, R. L. and Goodstein, D. L., Phys. Rev. A 9 (1974) 2657 ;

Stewart, G. A., Siegel, S. and Goodstein, D. L., in Proceedings of the Thirteenth International Conference on Low Temperature Physics, R. H. Kropschot and K. D. Timmerhaus, eds. (Plenum, New York) 1974 ;

GreIF, J. M., Ph. D. thesis, California Institute of Technology (1982) (unpublished).

[41] Carneiro, K., Passell, L. S., Thomlinson, W. and TAUB, H., Phys. Rev. B 24 (1981) 1170 and references contained therein.

[42] Novaco, A. D., Phys. Rev. A 8 (1973) 3065.

[43] Campbell, C. E., Milford, F. J., Novaco, A. D. and Schick, M., Phys. Rev. A 6 (1972) 1648.

[44] Liu, K. S., Kalos, M. H. and Chester, G. V., Phys. Rev. B 13 (1976) 1971.

[45] This complex notation is used to make explicit several features of the bilayer. The area per atom in the first layer $\alpha_{1}$ may differ from $a_{1}$ because of the presence of the second-layer fluid; $\alpha_{2}$ is the area per atom of the second-layer fluid; $\varepsilon_{0}$ is the single adatom binding to the substrate; in the numbering system of selective adsorption experiments $\varepsilon_{2}$ is the second excited state of the adatom in the holding potential; $v_{2}\left(\alpha_{1}\right)$ is the generalization of $\varepsilon_{\mathrm{II}}+\Phi_{12}$, with the dependence on the firstlayer density noted explicitly.

[46] KRIM, J. and DASH, J. G. (to be published);

Migone, A., Krim, J., Dash, J. G. and Suzanne, J. (to be published).

[47] RichaRDS, M. G. (private communication).

[48] Crawford, R. K., in Rare Gas Solids, Vol. II, M. L. Klein and J. A. Venables, eds. (Academic Press, New York) 1977.

[49] TAUB, H. (private communication).

[50] Allen, R. E. and De Wette, F. W., Phys. Rev. 179 (1969) 873.

[51] NI, X.-Z. and Bruch, L. W. (to be published).

[52] Hirschfelder, J. O., Curtiss, C. F. and Bird, R. B., The Molecular Theory of Gases and Liquids (Wiley, New York) 1964.

[53] Glachant, A. and Bienfait, M. (private communication).

[54] Glachant, A. and Bardi, U., Surface Sci. 87 (1979) 187. 